\title{
Antioxidant therapy on ischemic hepatitis: here we are and where do we go?
}

\author{
Hitoshi Maruyama ${ }^{1} \cdot$ Shuichiro Shiina ${ }^{1}$
}

Received: 23 February 2020 / Accepted: 7 April 2020 / Published online: 29 April 2020

(c) Asian Pacific Association for the Study of the Liver 2020

Keywords Ischemic hepatitis $\cdot N$-acetylcysteine $\cdot$ Acute liver failure $\cdot$ Acute kidney injury

$\begin{array}{ll}\text { Abbreviations } \\ \text { IH } & \text { Ischemic hepatitis } \\ \text { AST } & \text { Aspartate aminotransferase } \\ \text { ALT } & \text { Alanine aminotransferase } \\ \text { ALF } & \text { Acute liver failure } \\ \text { HE } & \text { Hepatic encephalopathy } \\ \text { ROS } & \text { Reactive oxygen species } \\ \text { NAC } & N \text {-Acetylcysteine } \\ \text { GSH } & \text { Glutathione } \\ \text { OR } & \text { Odds ratio } \\ \text { AKI } & \text { Acute kidney injury }\end{array}$

Ischemic hepatitis (IH) is a clinical manifestation caused by the insufficiency of hepatic flow volume and/or oxygen content to maintain hepatocytes. IH, hypoxic hepatitis and shock liver are in the same entity characterized by hepatic necrosis around the central veins, showing a transient, rapid, and considerable increase in liver enzymes (aspartate aminotransferase [AST] and alanine aminotransferase [ALT]) and significantly influences the prognosis [1-3]. It accounts for acute liver failure (ALF), which is a sudden and severe hepatic injury defined by an international normalized ratio $\geq 1.5$, neurologic dysfunction with any degree of hepatic encephalopathy (HE), no prior evidence of liver disease, and disease course of $\leq 26$ weeks [4]. A recent multicenter study of 387 patients in the US reported 4 common etiologies of ALF: IH (42.7\%), pancreatobiliary causes $(12.3 \%)$, drug-induced liver injury (11.3\%), and acute viral hepatitis (11.3\%) [5]. When the data were analyzed in patients with an ALT level > 5000, acetaminophenrelated liver injury and IH were the main causes $(55.6 \%$

Hitoshi Maruyama

maru-cib@umin.ac.jp

1 Department of Gastroenterology, Juntendo University, 2-1-1, Hongo, Bunkyo-ku, Tokyo 113-8421, Japan and $33.3 \%$, respectively). Similarly, in patients with an AST level $>5000$, IH and acetaminophen-related liver injury were also the main causes ( $60.3 \%$ and $25.9 \%$, respectively).

A large-scale systematic review of 24 papers including 1782 patients reported that IH was present in $2.5 \%$ of intensive care unit admissions, and $57 \%$ of patients with liver enzymes $>1000 \mathrm{IU} / \mathrm{L}$ had IH.[1]. The vast majority of patients with IH had cardiac comorbidities leading to congestive hepatopathy. Systemic hypotension may be a major pathogenesis of $\mathrm{IH}$, although the pooled proportion of patients with documented hypotension was only $52.9 \%$ [1]. In hepatology, acute variceal bleeding is a common cause of IH in patients with cirrhosis (Fig. 1), known as a medical emergency associated with a $20 \%$ mortality at 6 weeks [6]. Despite recent improvements in the quality of medical care, $\mathrm{IH}$ is a critical condition that requires prompt and proper treatment because the pooled rate of survival at discharge was $51 \%$ (range $23.1-85.7 \%$ ) [1].

Reactive oxygen species (ROS) production is a principal pathophysiology in $\mathrm{IH}$; ischemic cell damage leads to intracellular oxidant stress, and proinflammatory cytokines, such as TNF- $\alpha$, IL- 1 , and interferon- $\gamma$ in hepatocytes play a role in producing ROS. Ischemia also activates Kupffer cell, which is the main source of ROS during the reperfusion period [7]. In addition, mitochondria are the major intracellular source of ROS by the production with cellular respiration.

$\mathrm{N}$-acetylcysteine (NAC) is $\mathrm{N}$-acetyl derivative of the natural amino acid L-cysteine with the following: (i) direct antioxidant activity, (ii) indirect antioxidant activity due to a precursor of glutathione (GSH, the main endogenous nucleophilic peptide that reacts with and neutralizes electrophilic and hence damaging molecules such as $\mathrm{N}$-acetylp-benzoquinone imine, the electrophilic metabolite of acetaminophen), (iii) enhancing effect of hepatosplanchnic flow and function and (iv) breaking activity of disulfides $[8,9]$. NAC has been widely used as a mucolytic agent 


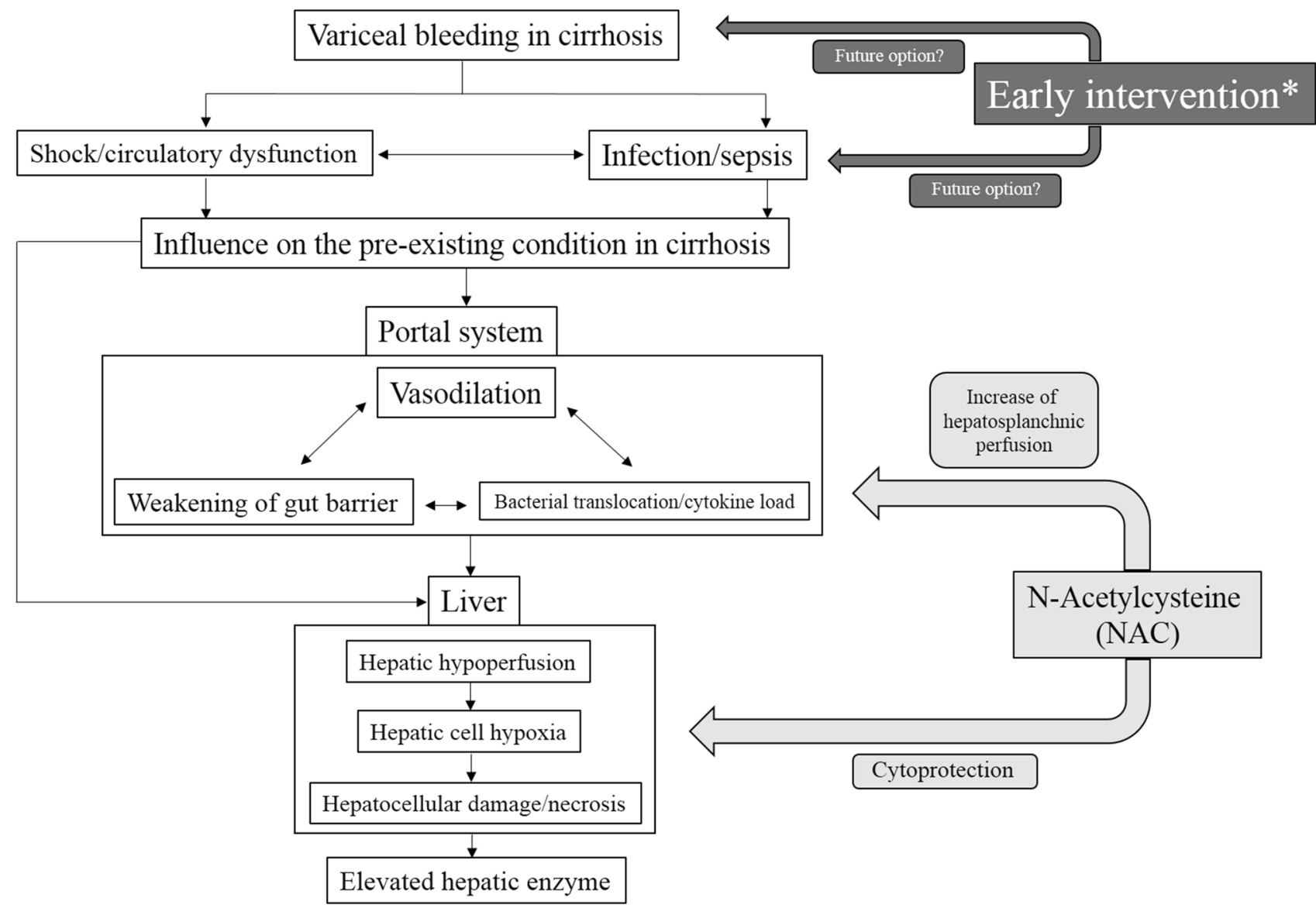

Fig. 1 Concept diagram of ischemic hepatitis following variceal bleed. Increase of hepatosplanchnic perfusion and cytoprotection against free-radical (oxidant) cellular damage are considered mechanisms to reduce hepatic ischemic injury by $\mathrm{N}$-acetylcysteine (NAC).

*Effect of early intervention (early administration of NAC, improvement of management for variceal bleeding and post-bleeding status) is expected as a potential future new option

and for the treatment of acetaminophen poisoning. The immediate administration of NAC is highly recommended in confirmed cases of acetaminophen toxicity; it is best administered within $8 \mathrm{~h}$ of ingestion [10] and is effective up to $48 \mathrm{~h}$ post-ingestion [11]. An oral NAC (loading dose: $140 \mathrm{mg} / \mathrm{kg}$ by mouth or as a $5 \%$ diluted solution through a nasogastric tube, maintenance dose: $70 \mathrm{mg} / \mathrm{kg}$ every $4 \mathrm{~h}$ for a total of 17 doses) is as effective as intravenous NAC (loading dose: $150 \mathrm{mg} / \mathrm{kg}$ in $5 \%$ dextrose solution over $15 \mathrm{~min}$, maintenance dose: $50 \mathrm{mg} / \mathrm{kg}$ given over $4 \mathrm{~h}$, followed by $100 \mathrm{mg} / \mathrm{kg}$ over $16 \mathrm{~h}$ ) and comparatively much less expensive [12]. However, the latter is more frequently used because most patients with acetaminophen-induced hepatotoxicity experience significant nausea and vomiting. Besides, NAC has side effects, mainly nausea and vomiting, while rash, urticaria, and bronchospasm rarely occur [13]. Even in cases without such side effects, altered mental status due to acetaminophen-induced conditions make it impossible to administer NAC orally [14].

NAC can be superior to placebo/supportive treatment, dimercaprol, and cysteamine in patients with acetaminophen overdose [15]. NAC may also reduce mortality in patients with fulminant hepatic failure caused by acetaminophen overdose (Peto odds ratio [OR] 0.26) [15]. In a randomized clinical trial comparing NAC with placebo in adults with non-acetaminophen ALF, NAC was associated with an improvement in transplant-free survival in a subgroup of patients with grade 1 and grade $2 \mathrm{HE}$ [16]. The positive effect of NAC on heat injury-mediated IH to reduce reperfusion injury was also reported [17]. These data suggest that NAC therapy should be considered early in the course of IH to improve transplant-free outcomes.

However, disagreements persist, as a recent systematic review demonstrated no difference in the overall survival at 3 weeks between NAC (70\%) and placebo (66\%), although NAC significantly improved the transplant-free survival compared with placebo: $40 \%$ NAC vs $27 \%$ placebo [18]. An etiology-based subgroup analysis also detected similar overall survival rates between the 
groups, NAC (79\%) vs placebo (65\%) with OR 0.50, despite improved transplant-free survival in patients with non-paracetamol drug-induced liver injury, NAC (58\%) vs placebo (27\%) with OR 0.27 . Therefore, there is insufficient evidence to support the effect of NAC in this regard, and further investigation is warranted.

Against this background, in a study conducted in India, Maiwall et al. reported interesting results regarding the effect of NAC on IH in cirrhosis patients accompanied by acute variceal bleeding [19]. The strength of this work was a prospective open-label randomized controlled trial with two groups (standard of care plus NAC or a placebo group) including a relatively large sample size of 220 patients. Furthermore, the subjects had various etiologies (cirrhosis mainly caused by alcohol abuse, and the others by viral, autoimmune, non-alcoholic steatohepatitis, or cryptogenic), not limited to drug-induced liver disease. The primary endpoint was the development of $\mathrm{IH}$ at day 5 , and the secondary end-points were survival at 6 weeks and the effect of NAC on other complications, HE, acute kidney injury (AKI), and infections (spontaneous bacterial peritonitis, pneumonia, urinary tract infection, and/or bacteremia) at day 5 . The study demonstrated two major points: lower deaths due to liver failure and reduced incidence of AKI. That is, the incidence of AKI was the only factor that showed a significant difference $(p=0.007)$ in the 5-day conditions; however, the study demonstrated no data explaining the kidney mechanism, as the authors acknowledged.

As with IH, ROS introduced by hypoxia and/or ischemia is a key factor in the pathogenesis of AKI, because the kidneys are highly sensitive to the impaired conditions [20]. In fact, the development of AKI indicates a close relationship with oxidative damage to tubular cells and renal tissue [21], as supported by animal studies demonstrating increased oxidative damage and decreased tissue antioxidant [22, 23]. Additionally, there is a linkage between uremia and increased circulating carbonyl and indole compounds, leading to enhanced systemic oxidative stress [24].

The application of antioxidant compounds has been used to treat AKI. Studies have shown the beneficial effects of NAC, being protective in ischemic, nephrotoxic, and rhabdomyolysis-induced AKI in animal models [25], improvement of kidney function, renal GSH, and systemic oxidative stress, and reduction of renal inflammation [20]. A recent animal study reported the possibility of molybdenum-based polyoxometalate nanoclusters with preferential renal uptake as novel nano-antioxidants for kidney protection; polyoxometalate nanoclusters efficiently alleviated clinical symptoms in mice subjected to AKI, as verified by dynamic positron emission tomography imaging with $68 \mathrm{Ga}$-ethylenediaminetetraacetate, serum tests, kidney tissue staining, and biomarker detection in the kidneys [26]. Taken together, the use of antioxidants seems to play a significant role against AKI irrespective of the presence/absence of $\mathrm{IH}$, and the benefits of NAC for IH might be dependent on the effect for AKI.

The data reported by Maiwall et al. may enhance the high expectations of NAC in the clinical application for IH [19]. However, unlike the favorable effect to the kidneys, their study reported no significant effect of NAC to control HE and infections, which are also considerable complications of IH in cirrhosis. That is, the use of NAC may not be a sole therapeutic strategy, but just one of the multidisciplinary treatments of IH. As future directions, firstly, it should be an initial step to predict the occurrence of IH by an intensive surveillance for those who have high-risk factors. Secondly, the effect of early interventions, including an early administration of NAC, and the improved management for variceal bleeding and post-bleeding status typified by shock and infections need to be examined as the next potential options for IH. Anyhow, the wide range of clinical presentations may feature treatment-resistive aspects of $\mathrm{IH}$, and the study by Maiwall et al. provide a rationale to pursue better therapies for IH in cirrhosis patients.

Funding None.

\section{Compliance with ethical standards}

Conflict of interest Hitoshi Maruyama and Shuichiro Shiina have declared that there is no conflict of interest.

Ethical approval This article does not contain any studies with human participants or animals performed by any of authors.

Informed consent Not necessary, see above.

\section{References}

1. Tapper EB, Sengupta N, Bonder A. The incidence and outcomes of ischemic hepatitis: a systematic review with meta-analysis. Am J Med. 2015;128:1314-1321.

2. Henrion J. Hypoxic hepatitis. Liver Int. 2012;32:1039-1052.

3. Waseem N, Chen PH. Hypoxic hepatitis: a review and clinical update. J Clin Transl Hepatol. 2016;4:263-268.

4. Flamm SL, Yang YX, Singh S, et al. American Gastroenterological Association Institute Guidelines for the Diagnosis and Management of Acute Liver Failure. Gastroenterology. 2017;152:644-647.

5. Breu AC, Patwardhan VR, Nayor J, et al. A mlticenter sudy ito cuses of svere aute lver ijury. Clin Gastroenterol Hepatol. 2019;17:1201-1203.

6. de Franchis R. Evolving consensus in portal hypertension report of the Baveno IV consensus workshop on methodology of diagnosis and therapy in portal hypertension. J Hepatol. 2005;43:167-176.

7. Muriel P. Role of free radicals in liver diseases. Hepatol Int. 2009;3:526-536. 
8. Aldini G, Altomare A, Baron G, et al. $N$-acetylcysteine as an antioxidant and disulphide breaking agent: the reasons why. Free Radic Res. 2018;52:751-562.

9. Rank N, Michel C, Haertel C, Lenhart A, Welte M, Meier-Hellmann A, Spies C. $N$-acetylcysteine increases liver blood flow and improves liver function in septic shock patients: results of a prospective, randomized, double-blind study. Crit Care Med. 2000;28:3799-3807.

10. Whyte IM, Francis B, Dawson AH. Safety and efficacy of intravenous $\mathrm{N}$-acetylcysteine for acetaminophen overdose: analysis of the Hunter Area Toxicology Service (HATS) database. Curr Med Res Opin. 2007;23:2359-2368.

11. Keays R, Harrison PM, Wendon JA, et al. Intravenous acetylcysteine in paracetamol induced fulminant hepatic failure: a prospective controlled trial. BMJ. 1991;303:1026-1029.

12. Kanter MZ. Comparison of oral and i.v. acetylcysteine in the treatment of acetaminophen poisoning. Am J Health Syst Pharm. 2006;63:1821-1827.

13. Sansone RA, Sansone LA. Getting a knack for NAC: $N$-acetylcysteine. Innov Clin Neurosci. 2011;8:10-14.

14. Montrief T, Koyfman A, Long B. Acute liver failure: a review for emergency physicians. Am J Emerg Med. 2019;37:329-337.

15. Brok J, Buckley $\mathbf{N}$, Gluud C. Interventions for paracetamol (acetaminophen) overdose. Cochrane Database Syst Rev. 2006;19:CD003328.

16. Lee WM, Hynan LS, Rossaro L, et al. Intravenous NAC improves transplant-free survival in early stage non-acetaminophen acute liver failure. Gastroenterology. 2009;137:856-864.

17. Will JS, Snyder CJ, Westerfield KL. $N$-acetylcysteine (NAC) for the prevention of liver failure in heat injury-mediated ischemic hepatitis. Mil Med. 2019;184:565-567.
18. Chughlay MF, Kramer N, Spearman CW, et al. $N$-acetylcysteine for non-paracetamol drug-induced liver injury: a systematic review. Br J Clin Pharmacol. 2016;81:1021-1029.

19. Maiwall R, Kumar A, Bhadoria AS, et al. Utility of $N$-acetylcysteine in ischemic hepatitis in cirrhotics with acute variceal bleed: a randomized controlled trial. Hepatol Int. 2020. https:// doi.org/10.1007/s12072-020-10013-5.

20. McCord JM. Oxygen-derived free radicals in postischemic tissue injury. N Engl J Med. 1985;312:159-163.

21. Dennis JM, Witting PK. Protective role for antioxidants in acute kidney disease. Nutrients. 2017;9:E718.

22. Paller MS, Hoidal JR, Ferris TF. Oxygen free radicals in ischemic acute renal failure in the rat. J Clin Investig. 1984;74:1156-1164.

23. Baliga R, Ueda N, Walker PD, et al. Oxidant mechanisms in toxic acute renal failure. Drug Metab Rev. 1999;31:971-997.

24. Himmelfarb J, Stenvinkel P, Ikizler TA, et al. The elephant in uremia: oxidant stress as a unifying concept of cardiovascular disease in uremia. Kidney Int. 2002;62:1524-1538.

25. Panizo N, Rubio-Navarro A, Amaro-Villalobos JM, et al. Molecular mechanisms and novel therapeutic approaches to rhabdomyolysis-induced acute kidney injury. Kidney Blood Press Res. 2015;40:520-532.

26. Ni D, Jiang D, Kutyreff CJ, et al. Molybdenum-based nanoclusters act as antioxidants and ameliorate acute kidney injury in mice. Nat Commun. 2018;9:5421.

Publisher's Note Springer Nature remains neutral with regard to jurisdictional claims in published maps and institutional affiliations. 